\title{
Interactive comment on "Evaluating the potential for Archaea to serve as ice nucleating particles" by Jessie M. Creamean et al.
}

\section{Anonymous Referee \#1}

Received and published: 30 November 2020

\section{General comments:}

Creamean at al. studied ice nucleation activity within the domain Archaea and are the first to report ice nucleation activity of this domain. Up to date, Archaea have not been evaluated as INP. In two out of four investigated species, the ability to induce freezing above $-18^{\circ} \mathrm{C}$ was found. The authors performed additional experiments (heat treatment, peroxide digestions) to further study the composition of INPs from Archaea. They suggest that the IN activity of intact cells were driven by organic and heat-labile materials.

This work provides valuable insights into ice nucleation activity within the domain Archaea and contributes to the understanding of biological ice nucleation as a whole. The results are certainly publishable and are aligned with the scope of the journal. 
Overall, the manuscript is well-written and -structured, and I recommend to publish it in Biogeosciences after the following comments have been addressed.

Specific comments:

Line 111: Where did you obtain the cell cultures from? Please provide more information.

Line 117: How did you determine the salinities?

Line 122, Table 1, Table 2: Usually ppt is understood as "parts per trillion". In specific disciplines such as oceanography, however, the abbreviation ppt is commonly used for "parts per thousand". I would recommend additional annotations to minimize misunderstandings.

Line 137: What is the temperature uncertainty of the measurement?

Line 138: If the freezing of wells was recorded in $0.5^{\circ} \mathrm{C}$ steps, how did you receive initial freezing temperatures such as $-17.6^{\circ} \mathrm{C}$ or $-19.2^{\circ} \mathrm{C}$ (lines $\left.152-153\right)$ ? Why do you have often more than two data points within one degree in all figures?

Line 140: How many independent experiments have been performed? Have the results been checked for reproducibility? Are the data presented in the figures arithmetic mean values? Otherwise, how did you calculate the standard error?

Line 142: For simplification, I would recommend to use the terms "heat" and "peroxide" (treatment) throughout the whole manuscript as they are also used in the figure legends.

Line 172: Is there any proof that the intact cells of $\mathrm{H}$. walsbyi and $\mathrm{N}$. pharaonis would show ice nucleation ability, which can be suppressed by lysis? You could additionally test if the ice nucleation ability of $\mathrm{H}$. morrhuae and $\mathrm{H}$. sulfurifontis can be suppressed upon lysis.

Lines 177, 180-182, 187-188: Which temperature do you refer to? Initial freezing 
Lines 182-186: Have you tried to use an excess of peroxide to remove all organic material, which could serve as INP, so that there are no differences between sample concentrations anymore?

Lines 187-188: Please refer to Figure 3 here.

Lines 190-191: Please clarify: "Interestingly, H. sulfurifontis INP spectra were more responsive to the heat treatment as opposed to peroxide, ...". Assuming that the same symbols and gray colors were chosen for figures 2 and 3 (legend is missing), the peroxide treatment shows a stronger reduction of INP spectra than the heat treatment.

Line 197: Can you really negate freezing point depression or is it only a diminution?

Table 1: The word "initial" can be confusing here as it describes the cell concentrations after culturing. Please optimize caption and footnote.

Table 2: I would recommend to explain "n/a" in a footnote rather than in the caption.

Fig. 1: Why do you have sometimes the same frozen fraction for different temperatures (also in the other figures)? I would recommend to show only changes in the frozen fraction. Please explain.

Fig. 2: You describe the plain lines in panel (b) as spectra for media controls, which underwent the heat and peroxide treatments. I can only see one plain line in panel (b) additional to the sample spectrum, but two more dashed lines, which have not been described in the caption yet. Please clarify the different lines. I would also recommend to use symbols instead of only colors in the legend. In the legend, you write "peroxide" but in the caption you name it "H2O2". Please be consistent. What kind of error bars did you use here? How do you explain that the heat-treated medium control shows a higher IN activity compared to the unamended control (panel b)?

Fig. 3: Please add a legend to the figure. Please also consider additional comments

Printer-friendly version

Discussion paper 
of Fig. 2. In this figure, it is the other way around, the peroxide-treated medium control shows a higher IN activity compared to the unamended control. Please explain.

It would be beneficial for the community to calculate an INP concentration using Vali's equation and compare the results to other biological INP.

Interactive comment on Biogeosciences Discuss., https://doi.org/10.5194/bg-2020-367, 2020. 